\title{
Ground Station Network Optimization for Space-to-Ground Optical Communication Links
}

\author{
Christian Fuchs and Florian Moll
}

\begin{abstract}
Space-to-ground optical data links enable higher data rates, require less electrical power, and allow more compact system designs than their corresponding RF counterparts. They may be applied to e.g. downlink earth observation data from low Earth orbit satellites, or as so-called feeder links for data transmission to geostationary telecommunication or multimedia satellites. However, optical space-to-ground links suffer from limited availability due to cloud blockage. The application of optical ground station (OGS) diversity and thus a network of OGS is required to overcome this limitation.

In this paper, we report on OGS networks and the calculation of combined network availabilities. Five years of cloud data gathered by a METEOSAT 2nd generation satellite have been evaluated. Single and joint site statistics as well as correlation between OGS sites are introduced. In order to effectively reduce computational effort, a network optimization method, exploiting correlation between sites and single-site availabilities, is proposed. Furthermore, the cloud data are used to find several optimal OGS networks and to simulate the networks' availabilities and temporal behaviors.

Optimal German, European and intercontinental networks are identified. With the increasing number of stations, the German network converges to an availability of $84.7 \%$, and the European network to around $99.9 \%$. The intercontinental network even reached an availability of $100 \%$ for 9 or more stations during the considered time span.
\end{abstract}

Index Terms-Optical Ground Station Diversity, Space-toGround Optical Communications

\section{INTRODUCTION}

O PTICAL communications for applications such as aircraft and satellite links gather increasing attention [1][4]. The high data rates and small terminal sizes, along with the fact that no licensing is required, make optical links a good choice for many applications. The first operational systems are starting to make use of this technology, as e.g. in the European Data Relay System [5].

Applications with very high capacity requirements, such as feeder links for geostationary telecommunication or multimedia satellites, in particular, can benefit from optical communication technology. Data rates of over 1 Tbps have already been demonstrated in short range horizontal links by using wavelength division multiplex (WDM) as done in [6], [7]. WDM would likely also be used in a Tbps GEO-ground system. However, as these applications require very high availabilities, link blockage by clouds cannot be tolerated and

Christian Fuchs and Florian Moll are with the Institute of Communications and Navigation, German Aerospace Center (DLR), Oberpfaffenhofen/Germany. E-mail: christian.fuchs@dlr.de. availabilities close to $100 \%$ must be achieved. To overcome this challenge, optical ground station diversity must be used.

Systems based on traditional RF technology make use of multiple ground stations mainly for the purpose of reusing spectrum. RF systems operating in $\mathrm{Q} / \mathrm{V}$ band or at even higher frequencies, however, may be affected by water clouds as well, thus requiring ground station diversity as well.

For very high system capacities, fewer ground stations may be required for systems with optical links than with RF links [8]. In order to assess practical availabilities of such ground station networks, remote sensing data from Earth observation satellites, especially in GEO, are very valuable. These data cover one shot measurements of large areas from which time synchronized samples for the individual locations can be extracted.

Clouds in the line of sight of a tropospheric propagation path can block the laser beam due to exorbitantly high extinction. Water clouds may have attenuations as high as 100 up to $600 \mathrm{~dB} / \mathrm{km}$ and even the thinnest exceed $30 \mathrm{~dB}$ of total attenuation [9], [10]. Ice clouds, however, attenuate less. Typical values are $1-6 \mathrm{~dB} / \mathrm{km}$ specific and $1-15 \mathrm{~dB}$ total attenuation [11]. Therefore, clouds either completely block the link or a link margin must deal with up to $15 \mathrm{~dB}$ attenuation when at least ice clouds shall be overcome. Overcoming water clouds is questionable, even if they are thin.

Eventually, water clouds are considered to cause an interruption of the communication link, ice clouds might be regarded in a link budget. For that reason, an investigation of cloud cover statistics of particular sites of interest must be performed in the planning and design phase of a satelliteground communication system. This system may comprise a single ground station or a set of ground stations. However, in the majority of the scenarios, diversity concepts based on multiple ground stations are under investigation to achieve a useful overall system availability. Corresponding studies are e.g. available for Europe [9], [12], USA [13]-[15], and Japan [16], [17].

Most often, passive remote sensing data are applied since they are widely available. Several globally distributed sites were assessed for a world-spanning ground station network for Deep Space and Moon links by analyzing GEO satellite images from GOES-West (Geostationary Operational Environmental Satellite-West), GOES-East, GOES-9, Meteosat-7 (Meteorological Satellite-7), and Meteosat-5 [13]. An optimization algorithm was applied here to reduce computational effort. The same database and algorithm in combination with images from the GEO MTSAT (Multi-Functional Transport 
Satellite) were applied to calculate worldwide distributed ground stations and network availability for LEO downlinks and Deep Space links [14]. MTSAT images were also used to simulate availability of LEO downlinks to the Japanese region [16], [17]. LEO satellite measurements were used in [9], [10] to determine HAP (High Altitude Platform)-to-ground link availability. MSG (Meteosat Second Generation) and MetOp (Meteorological Operational Satellite) images were used in [12] for analysis of satellite downlink availability with ground stations distributed over Europe and northern Africa. An optimization algorithm was also applied to find the optimum network. This algorithm was compared to a newer one presented in [18] and superiority of the later one was demonstrated.

Remote active measurements are available from the LEO LIDAR satellite system CALYPSO (Cloud-Aerosol Lidar and Infrared Pathfinder Satellite Observation). These were used together with the passive measurements from MSG in [19] to derive high cloud attenuation with co-aligned LIDAR/camera data for feeder links served by ground stations in Europe. To date, LIDAR data are only useful for monthly or seasonal statistics since sampling by the LIDAR scan is very low as discussed in [20]. Ground observation databases are also commonly used, as e.g. in [15] to estimate single and joint site availabilities, here for a Deep Space link, and in [21] for a Moon-Ground link.

In our paper, we investigate the availability of an optical GEO feeder link with a GEO located over Europe. The objective is to determine efficient algorithms for deriving optimal or close to optimal OGS networks. Furthermore, the network behavior is analyzed in terms of availability and statistics of outage duration, with increasing numbers of stations. Three networks with different spread (German, European, intercontinental) are considered.

We use MSG data with a temporal resolution of $15 \mathrm{~min}$. This gives an excellent statistical basis in order to determine the best ground station network with lowest overall probability of link loss. A new optimization algorithm is developed to effectively find an optimum network within the area of the measurement data, which is different from the ones already presented in [12], [13], [18], [19], [22]. Our selection algorithm makes use of the measured correlation coefficient of OGS locations, similar to the study for Japan in [17], however by considering correlation in the whole network, and in combination with the single site unavailability for preselection and reduction of the data base. Furthermore, investigation of time behavior is included to analyze the system with respect to service types that have specific demands on temporal performance, like real-time, near real-time, or delay-tolerant services. The presented techniques are especially applicable for optical links from geostationary satellites to ground, as the data for the simulations were taken with a GEO platform.

The structure of this paper is as follows: Section II describes the analytical calculation of OGS network availabilities with and without considering correlation between sites. Section III explains the methodology of the simulations and also describes the data evaluation as well as methods to optimize OGS networks. A method to derive optimal OGS networks based on single-site statistics as well as correlation between OGS sites is introduced. Section IV discusses the optimization results derived with the previously introduced methods. Furthermore, analysis results of availability and outage duration for three different networks (German, European and intercontinental) are given and discussed. Section V gives conclusions. Appendix A lists all OGS sites used for optimization within this study.

\section{Analytical Analysis of Network Availability}

\section{A. Definition of an OGS Network}

With $M$ being the availability requirement, i.e. the least number of ground stations that shall be available at any given time, $a$ being the number of ground stations actually being available at a given time, and $N$ being the number of stations in the network, the combined $M$-out-of- $N$-availability $A$ can be defined. Subsequently, a Link Outage Probability can be defined as $\mathrm{LOP}=1-A$.

A typical network definition includes the number of stations in the network (e.g. $N=8$ ) and an availability requirement (e.g. $M=1$, i.e. at least one station shall be available at any time). In the end, the availability $A$ results from $N$ and $M$.

\section{B. Uncorrelated OGS Networks}

1) OGS Networks with Arbitrary Availabilities: In the case of uncorrelated weather conditions between the OGS forming the network, i.e. the weather influences of all OGS are statistically uncorrelated, the combined availability of any given network can be calculated analytically based on singlesite availability statistics. The long-term single-site availability of a given OGS site is easily accessible, may it be based on local weather observations or on satellite data. However, the drawback of using these data sources is that correlation between sites is not considered.

$p_{i}$ is considered the availability of one ground station with index $i$, and $q_{i}=1-p_{i}$ is considered the non-availability. The availability $A$ is calculated as

$$
A=1-\mathrm{P}(0)-\mathrm{P}(1)-\cdots-\mathrm{P}(M-1)
$$

with $\mathrm{P}(a)$ being the probability that exactly $a$ stations are available at a given time. In other words, $\mathrm{P}(0)$ is the probability that no station is available, $\mathrm{P}(1)$ is the probability that exactly one station is available, and so on. These probabilities are subtracted from one $(=100 \%)$, resulting in the probability that at least $M$ stations are available. Thus, the probabilities $\mathrm{P}(0 \ldots M-1)$ have to be calculated.

The probability $\mathrm{P}(0)$, i.e. of having cloudy conditions at all ground stations, is the product over all non-availabilities $q_{i}$ :

$$
\mathrm{P}(0)=q_{1} q_{2} \cdots q_{N}
$$

Thus, the probability of having at least one single ground station available reduces to the expression

$$
\mathrm{P}(1)=1-\mathrm{P}(0)
$$

The calculation of $\mathrm{P}(a)$ for arbitrary values of $a$ is, however, more complicated, as all possible combinations of 
availability and cloudiness need to be evaluated. The number of possible combinations $\mathrm{N}_{\text {comb }}$ is given by the binomial coefficient of $N$ over $a$.

By evaluating all $\mathrm{N}_{\text {comb }}$ combinations, the probability of having exactly one out of four stations available can be calculated as

$$
\mathrm{P}(1)=p_{1} q_{2} q_{3} q_{4}+\cdots+p_{4} q_{1} q_{2} q_{3}
$$

In this manner, Equation 1 is evaluated and the combined availability of an OGS network can be calculated.

2) OGS Networks with Constant Availabilities: In the case of constant $p$, i.e. a ground station network with equal availabilities at all sites, the above equations can be simplified. Although this case is unlikely to appear in reality, it is included here to give an impression about the number and quality of sites required in order to reach a certain combined network availability. It can be seen as upper bound of network availability without the effect of weather correlation between sites.

With equal availabilities, Equation 4 simplifies to

$$
\mathrm{P}(1)=4 \cdot p \cdot q^{3}
$$

This leads to the generalized term

$$
\mathrm{P}(a, N)=\left(\begin{array}{c}
N \\
a
\end{array}\right) \cdot p^{a} \cdot q^{N-a}
$$

and allows to rewrite Equation 1 as:

$$
A=1-\sum_{a=0}^{M-1}\left(\begin{array}{c}
N \\
a
\end{array}\right) p^{a} q^{N-a}
$$

or

$$
\mathrm{LOP}=1-A=\sum_{a=0}^{M-1}\left(\begin{array}{c}
N \\
a
\end{array}\right) p^{a} q^{N-a}
$$

Figure 1 shows example results of Equation 8 vs. the number of stations in a network for $M=1,2,3$ available stations.

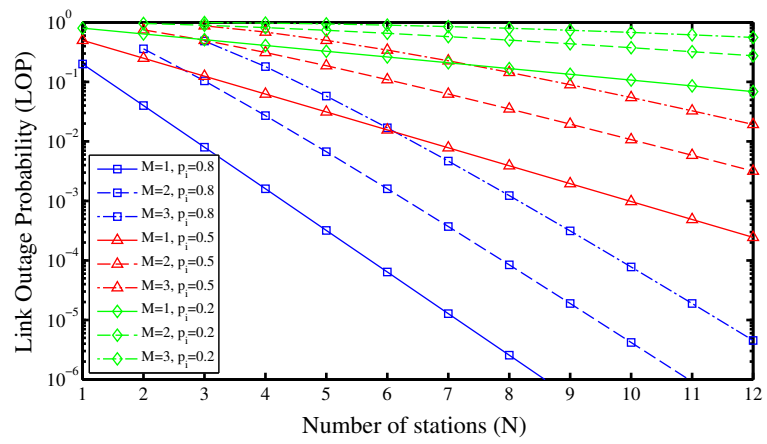

Fig. 1. Link Outage Probability (LOP) for multiple station availability $(M=$ $1,2,3$ ) for different single site availabilities $p$ vs. different numbers of stations $N$ in a network

It can be observed that about 4 OGS with an individual availability of $p_{i}=0.8$ are required to reach a Link Outage Probability of $10^{-3}$ for an availability requirement of $M=1$, while an OGS network with $p_{i}=0.5$ already requires about 10 sites to reach the same figure. Trivially, for a given LOP, the required number of stations increases as well if a higher availability requirement of e.g. $M=2$ or $M=3$ is given.

\section{Correlated OGS Networks}

If correlation is taken into account when calculating the availabilities of the ground stations forming an OGS network, the estimation of the combined network availability becomes more challenging.

Table I shows the correlation matrix of a network with $N$ stations and its correlation coefficients $r_{i, k}$. The correlation coefficient of a station with itself is 1, i.e. $r_{i, k}=1$ for $i=k$. The matrix is symmetric, hence $r_{i, k}=r_{k, i}$. For the theoretical case of an uncorrelated network, all $r_{i, k}$ with $i \neq k$ would equal to 0 .

\begin{tabular}{c|ccccc}
$i, k$ & 1 & 2 & 3 & $\ldots$ & $\mathrm{N}$ \\
\hline 1 & 1 & $r_{1,2}$ & $r_{1,3}$ & $\ldots$ & $r_{1, N}$ \\
2 & $r_{2,1}$ & 1 & $r_{2,3}$ & $\ldots$ & $r_{2, N}$ \\
3 & $r_{3,1}$ & $r_{3,2}$ & 1 & $\ldots$ & $r_{3, N}$ \\
$\ldots$ & $\ldots$ & $\cdots$ & $\ldots$ & $\ldots$ & $\cdots$ \\
$\mathrm{N}$ & $r_{N, 1}$ & $r_{N, 2}$ & $r_{N, 3}$ & $\ldots$ & 1
\end{tabular}

TABLE I

CORRELATION COEFFICIENTS FOR AN OGS NETWORK WITH $m=4$ STATIONS

We define the mean correlation of a network $\overline{r_{S}}$ as mean of the elements in the upper triangle of the correlation matrix, with $\left(\begin{array}{c}N \\ 2\end{array}\right)$ being the number of elements in the upper triangle:

$$
\overline{r_{S}}=\frac{1}{\left(\begin{array}{c}
N \\
2
\end{array}\right)} \cdot \sum_{i=1}^{N-1} \sum_{k=i+1}^{N} r_{i, k}
$$

Furthermore, we define the sum over each column without $i=k$ (i.e. excluding all ones) of the correlation matrix as mean correlation of a single station:

$$
\overline{r_{i}}=\frac{1}{N-1} \cdot \sum_{k=1}^{N, k \neq i} r_{i, k}
$$

Figure 2 shows a visual example of the correlation coefficients for 12 German ground station sites. The ground station indexes are listed in Table $\mathrm{V}$ of Appendix A. Red values indicate high correlation coefficients, green indicates a correlation coefficient of 0 . The numbers given in the centers of the squares show the distance between the corresponding ground station sites in $\mathrm{km}$. It can be seen that sites with large separation yield lower correlation.

\section{Simulation of Network Availability}

\section{A. Cloud Data}

A large variety of satellite data, suitable for the evaluation of OGS availabilities, are available. This includes data from LEO and GEO missions. Due to its high temporal and spatial resolution, as well as for the good coverage of the regions of interest, a Meteosat Second Generation (MSG) GEO satellite was chosen for the analysis within this study. The SEVIRI (Spinning Enhanced Visible and Infrared Imager) instrument [23] onboard acquires images with a resolution of $3712 \times 3712$ pixels, a time step of 15 minutes, and a spatial resolution of $3 \mathrm{~km}$ in NADIR direction.

The cloud mask product MSGCLMK offers cloud data on a binary basis, i.e. values are given for cloudy/non-cloud 


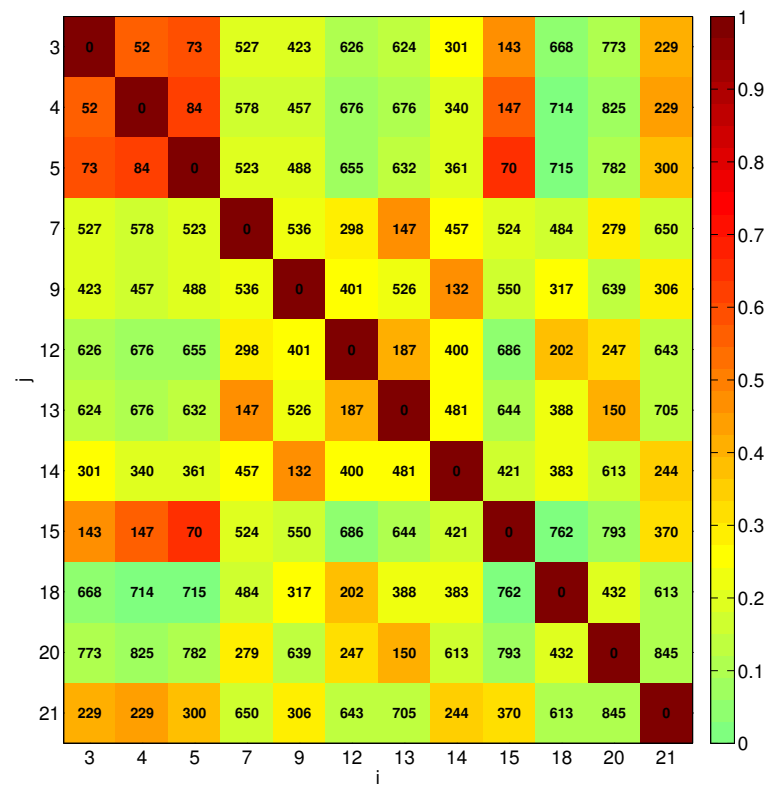

Fig. 2. Visual representation of correlation matrix for 12 sites in Germany. The numbers in the center of the colored squares show the distance between the two corresponding ground station sites. Red corresponds to a correlation coefficient of 1 .

conditions. A description of how cloud mask data are derived from SEVIRI data is available e.g. in [24], [25]. We assume that a "cloudy pixel" and a "clear sky pixel" correspond to a cloud having an attenuation that, respectively, cannot be overcome and can be overcome by a margin in the link budget.

Figure 3 shows an example image with MSG footprint projected on a world map. Europe as well as Africa and parts of South America are well-covered by the available data.

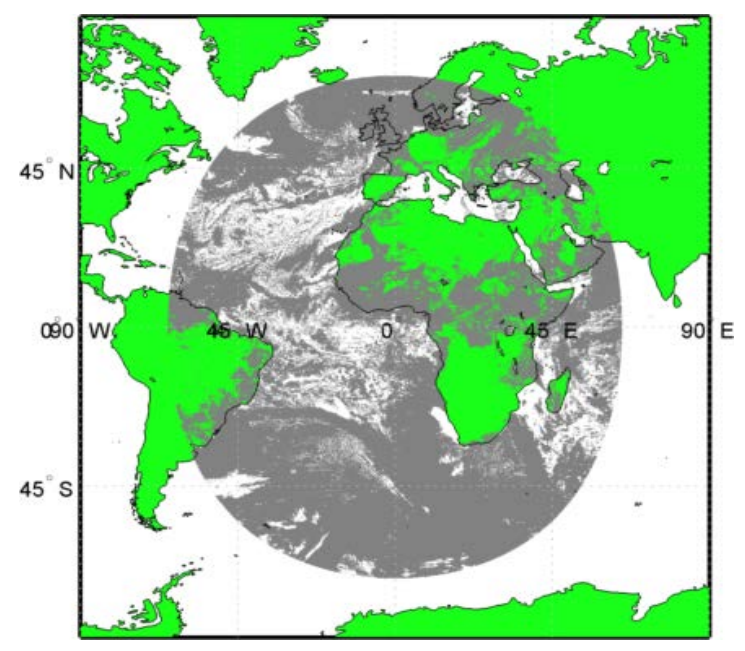

Fig. 3. Example SEVIRI full disk image acquired with Meteosat Second Generation 2 (August 1, 2012, 11:15 UTC). Gray pixels are clouds.

A total of five years of data (2008-2012) sampled at an interval of 15 minutes are used for all evaluations. Table II compares the theoretical number of data points with the number of points which were actually available. It can be observed that up to roughly $3 \%$ of data are missing due to malfunctions of SEVIRI, however, this can be easily tolerated by correspondingly adapting the evaluation algorithms.

\begin{tabular}{cccccc}
\hline & & \multicolumn{2}{c}{ Data Points } & \multicolumn{2}{c}{ Missing } \\
Year & Sampling & Theoretical & Actual & Total & $\%$ \\
\hline 2008 & $15 \mathrm{~min}$ & 35136 & 34116 & 1020 & 2.90 \\
2009 & $15 \mathrm{~min}$ & 35040 & 33868 & 1172 & 3.34 \\
2010 & $15 \mathrm{~min}$ & 35040 & 35012 & 28 & 0.08 \\
2011 & $15 \mathrm{~min}$ & 35040 & 35003 & 37 & 0.11 \\
2012 & $15 \mathrm{~min}$ & 35136 & 35095 & 41 & 0.12 \\
\hline $2008-2012$ & $15 \mathrm{~min}$ & 175392 & 173094 & 2298 & 1.31 \\
\hline
\end{tabular}

TABLE II

REVIEW OF AVAILABLE CLOUd DATA THAT WAS USED FOR THE EVALUATION

\section{B. Methodology}

1) Pre-Processing: Due to the high temporal and spatial resolution of the available images, a large amount of data needs to be processed. To reduce the time required for various evaluations, the data have been pre-processed by generating a database with a quantity of $\mathrm{N}_{\mathrm{db}}$ potential OGS sites within the visibility of MSG2. The sites have been selected according to criteria such as available infrastructure and political stability; the site selection was, however, not exhaustive, thus further sites might be added for future evaluations. The selected OGS are shown in Appendix A.

For each OGS, a vector $\mathbf{c}_{i}$ with binary cloud data $(0-$ no cloud, 1 - cloud) was generated. $\mathrm{N}_{\text {Samples }}$ is the number of available data points.

$$
\mathbf{c}_{i}=\left[c_{1}, c_{2}, \ldots c_{j}, \ldots c_{\mathrm{N}_{\text {Samples }}}\right]
$$

This allowed for a large data reduction and for simpler evaluation.

With site index $i$ and data point index $j$, a Matrix $\mathbf{C}$ can be defined. Each row in $\mathbf{C}$ holds the cloudiness data of one OGS in the network.

$$
\mathbf{C}=\left(\begin{array}{c}
\mathbf{c}_{1} \\
\mathbf{c}_{2} \\
\ldots \\
\mathbf{c}_{N}
\end{array}\right)
$$

A network of sites is defined by assigning rows of $\mathbf{C}$ corresponding to the desired station sites. For this study, a subset $S$ is used for the definition of a network. As an example, a four station network may be defined by $S=\{1,7,12,19\}$.

2) Calculation of Uncorrelated Availability: To calculate a network availability without taking the correlation between the sites into account, the availabilities of single stations have to be calculated. The combined (uncorrelated) availability can then be calculated as stated in Equation 3 .

The non-availability of a single station can be calculated by summing up all elements in the cloud vector for that station and by dividing the result through the number of available samples for the station:

$$
q_{i}=\frac{1}{N_{\text {Samples }}} \sum_{j=1}^{N_{\text {Samples }}} c_{i, j}
$$


By combining $p_{i}=1-q_{i}$ with Equation 3, the probability that at least one station is available in the network $S$ can be written as

$$
P(1)=1-\prod_{i}^{S} \frac{1}{N_{\text {Samples }}} \sum_{j=1}^{N_{\text {Samples }}} c_{i, j}
$$

3) Calculation of Correlated Availability: By summing up all data points for each time sample $j$, the quantity of unavailable stations can be calculated. The number of available stations $a$ for each $j$ can then be calculated by subtracting the result from the number of stations in the network $\mathrm{N}$ :

$$
a_{j}=\mathrm{N}-\sum_{i \in S} c_{i, j}
$$

With the availability requirement $M$,

$$
a_{j}^{*}=\left\{\begin{array}{l}
1, a_{j} \geq M \\
0, a_{j}<M
\end{array}\right.
$$

can be calculated for each $j$.

Analog to Equation 14, the availability of the network can be calculated as

$$
A=\frac{1}{N_{\text {Samples }}} \sum_{j=1}^{N_{\text {Samples }}} a_{j}^{*}
$$

\section{OGS Network Optimization}

1) ...by using single site availability (Method 1): The easiest method to find an optimal ground station network is to calculate the single site availabilities of all stations within the available database, and trivially select the stations with the highest availabilities. This approach does not consider correlation and does not take into account that two stations might be placed in close proximity, which would not result in increased availability due to the correlated weather conditions at both sites.

2) ...by calculating all possible combinations (Method 2): This method takes correlation into account. Given the preprocessing approach and having a reduced set of cloudiness data in $\mathbf{C}$, an m-out-of-n network can be optimized by creating a vector $v_{j}$ according to Equation 16 for each possible combination, and a subsequent maximization of the result. The number of combinations $\mathrm{N}_{\text {comb }}$ can get extremely high for large networks and/or station databases.

This approach works well for small networks and small site databases, however, optimizations for large databases are challenging, as many different combinations must be tested. To optimize a network with a database size of $N_{d b}=66$, as used within this study, and up to $N=12$ stations, about $10^{13}$ combinations would have to be tested for the total number of 173094 data points. This approach is inefficient and very time consuming.

3) ...by using site correlation and single site availability (Method 3): With Method 3, the correlation between ground stations as well as the single site availability is used to preselect potential ground station sites on the one hand, and to preselect potentially optimal combinations for calculating an optimal network on the other hand.
For the preselection of ground station sites, we use the stations' mean correlation coefficient $\overline{r_{i}}$ defined in Equation 10 and multiply it with the non-availability $q_{i}$ of the station as defined in Equation 13. We define the result as cloudinessparameter $r q_{i}$ :

$$
r q_{i}=\overline{r_{i}} \cdot q_{i}
$$

Ground station sites with a small $r q_{i}$ have little correlation to other sites and a low cloud probability. Within this study, we used this approach to preselect the $N_{r}=25$ station sites with the lowest $r q_{i}$ for the further investigations of each network.

Although this already dramatically decreases the required computational effort compared to optimization Method 2, as the number of stations is reduced to $25, \mathrm{~N}_{\text {comb }} \approx 5 \cdot 10^{6}$ operations on $\mathrm{N}_{\text {Samples }}=173094$ data points are still required for a full optimization of e.g. a 12 station network.

To further reduce the computational effort, potentially optimal combinations of station sites are preselected. For this, we multiply the network's mean correlation $\overline{r_{S}}$ with the uncorrelated network unavailability known from Equation 2:

$$
r_{q, S}=\overline{r_{S}} \cdot \prod_{i \in S} q_{i}
$$

With this metric, we select the $N_{q}$ smallest values of $r_{q, S}$ and thus the $N_{q}$ best combinations. $N_{q}=10^{5}$ has been selected for the further evaluations. A discussion of the influence of $N_{r}$ and $N_{q}$ on the network optimization process is available in section IV.

Finally, the network availability of all remaining $N_{q}$ combinations can be calculated, and the combination with highest availability is assumed to be the best OGS network.

\section{RESULTS}

\section{A. Network Optimization}

1) Overview: Three different networks have been considered: A network with stations in Germany only, a network with stations expanding to Europe, and an intercontinental network, also making use of stations in Africa, South-America, and the Near East. Table III shows which stations were used from the database in Appendix A.

\begin{tabular}{lcc}
\hline Network & Indexes & $\mathrm{N}_{\mathrm{db}}$ \\
\hline German & $1-22$ & 22 \\
European & $1-49$ & 49 \\
Intercontinental & $1-66$ & 66 \\
\hline
\end{tabular}

TABLE III

OPTICAL GROUND STATION SITES USED FOR THE OPTIMIZATION OF SEVERAL NETWORKS WITH DATABASE SIZE $\mathrm{N}_{\mathrm{dB}}$. INDEXES CORRESPOND TO THE TABLES IN APPENDIX A.

These three networks are used to investigate the previously defined optimization methods and to further analyze the statistical behavior of each network. 
2) German Network: An example optimization result, comparing the different optimization methods for the German network, is available in Table IV. Method 2 was only used for small $N$, as with larger networks the computation time becomes too large for evaluations within a reasonable timeframe. Comparing Method 1 and 2/3, it can be observed that the results are remarkably different, as Method 1 does not include correlation between ground station sites. This can be observed even for small networks with $N=2$, where Method 1 simply delivers the two stations with highest single site availability (4 and 6), while Methods 2 and 3 deliver different stations (4 and 18). The same can be observed for larger networks: Following Method 1, station number 3 is already added to the network at $N=4$, while with Method 3, it is not added before $N=8$. Furthermore, it can be observed that Methods 2 and 3 deliver the same results. This indicates that Method 3 can be used to reduce the computational effort and will deliver a result which is optimal or at least close to the optimal solution.

\begin{tabular}{llll}
\hline$N$ & Method 1 & Method 2 & Method 3 \\
\hline 1 & 4 & 4 & 4 \\
2 & 4,6 & 4,18 & 4,18 \\
3 & $4,6,5$ & $4,18,20$ & $4,18,20$ \\
4 & $4,6,5,3$ & $4,14,18,20$ & $4,14,18,20$ \\
5 & $4,6,5,3,1$ & - & $4,7,14,18,20$ \\
6 & $4,6,5,3,1,8$ & - & $4,7,14,18,20,21$ \\
7 & $4,6,5,3,1,8,2$ & - & $4,7,12,14,18,20,21$ \\
8 & $4,6,5,3,1,8,2,11$ & - & $3,4,7,14,15,18,20,21$ \\
\hline
\end{tabular}

TABLE IV

OPTIMIZATION RESULTS FOR A NETWORK WITH STATIONS IN GERMANY

Figure 4 shows the Link Outage Probability (LOP) for German networks with different numbers of stations, again comparing the different optimization methods. It can be observed that especially in small-scale networks, e.g. within Germany, the approaches with and without correlation yield different results. Without considering correlation, the LOP of an OGS network in Germany is remarkably underestimated.

Furthermore, it can be observed that the LOP with correlation (Method 3) is approaching a limit of about 0.1. Even by adding further stations in Germany, no considerable improvement is expected.

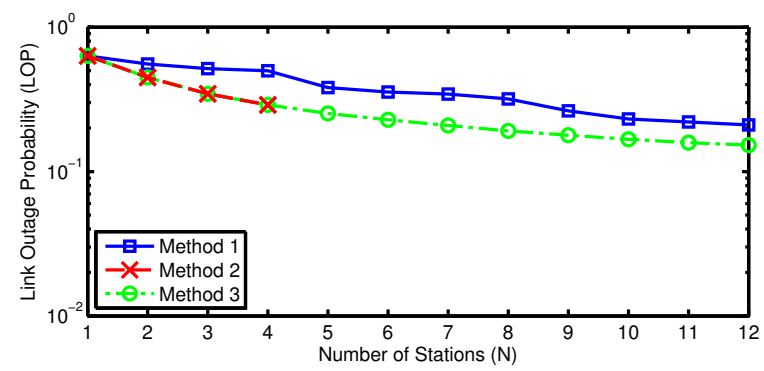

Fig. 4. Optimization results for 3 different methods (German network)

Figure 5 shows a map of the 12 best stations in Germany, according to optimization method 3.

3) European Network: Figure 6 shows the different optimization methods for stations selected from all over Europe.

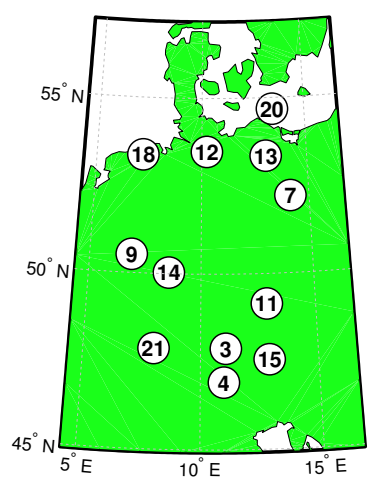

Fig. 5. Optimal station locations for a 12 station network in Germany. The indexes are available in Appendix A.

As in the German case, the results of Methods 1 and $2 / 3$ differ for an increasing number of stations within the network. This indicates that the best stations for the network still show considerable correlation.

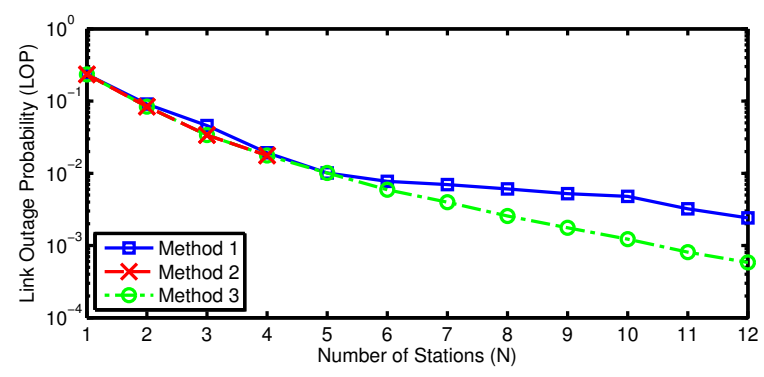

Fig. 6. Optimization results for 3 different methods (European network). The indexes are available in Appendix A.

Figure 7 shows a map of the 12 best stations in Europe, according to optimization method 3 .

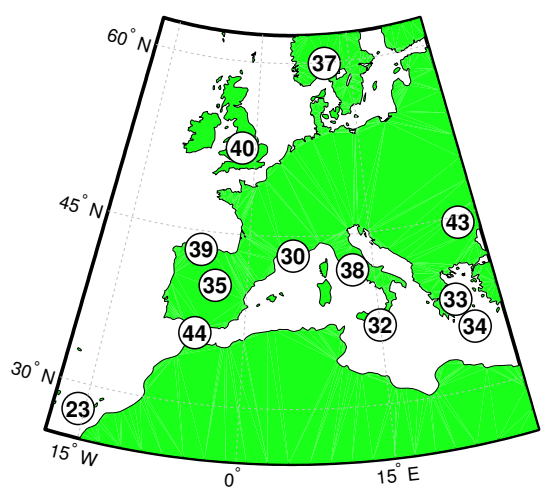

Fig. 7. Optimal station locations for a 12 station network in Europe. The indexes are available in Appendix A.

4) Intercontinental Network: In conclusion, Figure 8 shows the calculated LOPs for an optimized intercontinental network. For intercontinental networks with 9 or more stations, the LOP was found to be 0 during the observation period of 5 years. For a reasonable statistical analysis of LOPs in the order of $10^{-5}$ and below, more cloud data would be required. 


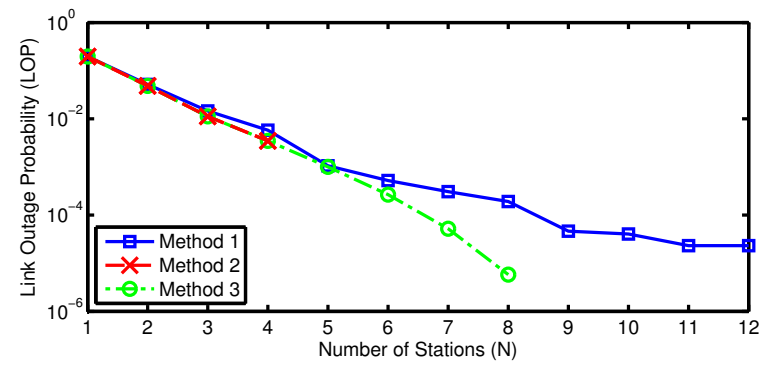

Fig. 8. Optimization results for 3 different methods (intercontinental network).

Figure 9 shows a map of the intercontinental stations that have been found to be optimal, according to optimization method 3 .

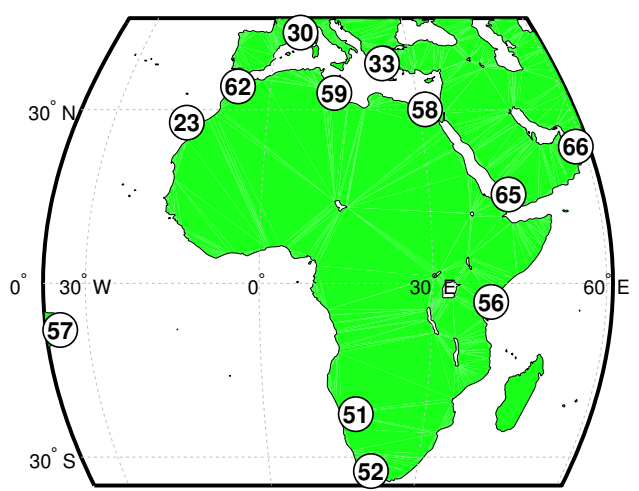

Fig. 9. Optimal station locations for a 12 station intercontinental network. The indexes are available in Appendix A.

5) Discussion of Method 3: As Method 3 makes use of preselection, it is sensitive to the number of preselected OGS sites $N_{r}$ as well as to the number of preselected OGS combinations $N_{q}$. In order to evaluate this sensitivity, network optimizations have been performed for all three networks with varying $N_{r}$ and $N_{q}$.

In Figure 10, $N_{r}$ is varied for a constant $N_{q}$ of $10^{5}$. It can be observed that Method 3 is indeed sensitive to the number of preselected sites $N_{q}$. Although for the small German network no difference is visible for the different values of $N_{q}$, the optimization results differ for the larger European and intercontinental networks, however, for $N_{r} \geq 20$, no relevant changes are visible.

Figure 11 shows exemplary optimization results for varying $N_{q}$ with a constant $N_{r}$ of 25 . It can be seen that Method 3 is also sensitive to the number of preselected combinations. Again, this fact is not apparent for the small German network, but can be observed for the larger European and intercontinental networks. The intercontinental network, for example, shows an interesting behavior for $N=8$. Here, a station combination yielding a better availability has not been included in the preselection with $N_{q}=10^{4}$, but has been present for $N_{q}=10^{5}$, decreasing the LOP from $1.7 \cdot 10^{-5}$ to $5.8 \cdot 10^{-6}$.

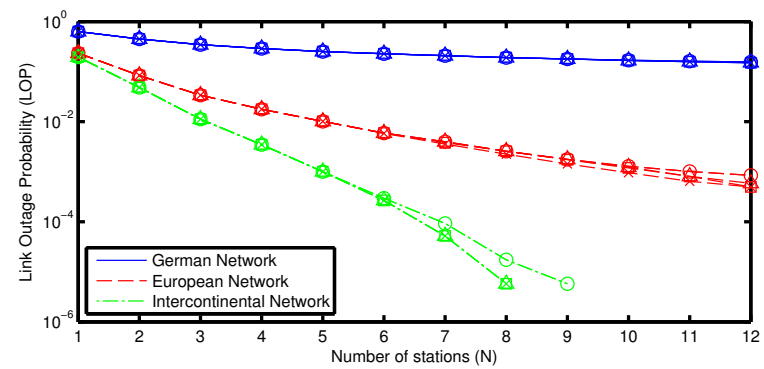

Fig. 10. LOP vs. number of stations $\mathrm{N}$ for different numbers of preselected OGS locations $N_{r}$ with constant number of preselected OGS combinations $N_{q}=10^{5}$. Square: $N_{r}=15$, triangle: $N_{r}=20$, Circle: $N_{r}=25$, Cross: $N_{r}=30$

Thus, it cannot be ensured, that the resulting OGS network is indeed optimal, but this approach with $N_{r}=25$ preselected OGS sites and $N_{q}=10^{5}$ preselected OGS combinations can be considered as good trade-off between computational speed and efficiency.

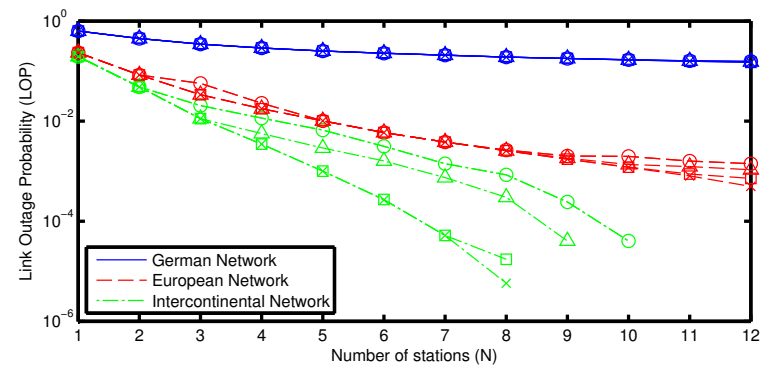

Fig. 11. LOP vs. number of stations for different numbers of preselected OGS combinations $N_{q}$ with constant number of preselected OGS locations $N_{r}=25$. Square: $N_{q}=10^{2}$, triangle: $N_{q}=10^{3}$, Circle: $N_{q}=10^{4}$, Cross: $N_{q}=10^{5}$.

\section{B. Network Availabilities}

Optimization method 3 is used for all further analysis. Figure 12 shows optimization results for networks with German, European, or intercontinental stations. The number of stations in the networks varied from 1 to 12 .

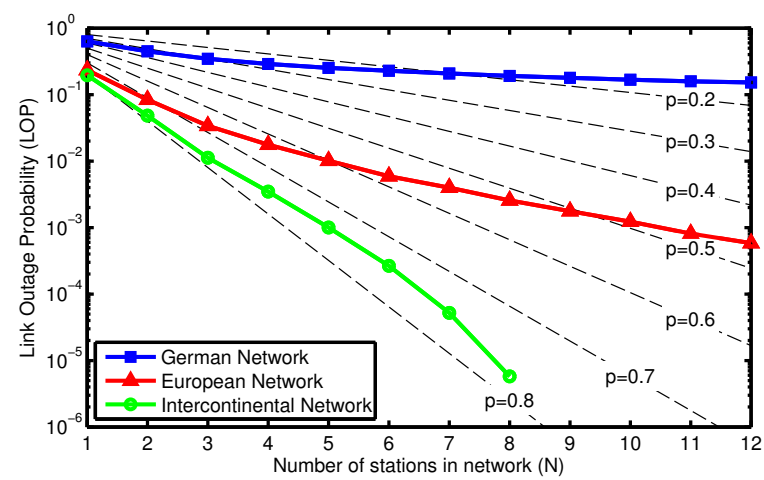

Fig. 12. Link Outage Probability (LOP) vs. number of stations $(N)$ for the three networks. The black dashed lines show the LOP of an uncorrelated network with $N$ stations for different single site availabilities $p$ (cp. Equation 8).

It can be observed that the German network only slightly benefits from an increasing number of stations. This is likely 
due to the fact, that the weather conditions within Germany do not allow sufficient decorrelation between the ground station sites.

For the European network, the availabilities of the single stations are higher, and, due to the fact that the distances between the ground stations are larger, the weather is suitably decorrelated and the Link Outage Probability decreases with an increasing number of stations.

For the intercontinental network, the situation becomes even better. No cloud blockage was detected within the 5year observation period for intercontinental networks with at least 10 stations. One likely reason for this is that the seasons between the Northern and Southern hemisphere are decorrelated. When it is winter in the Northern hemisphere and the conditions are worse for the ground stations located in the Mediterranean, it is summer in the Southern hemisphere. Conditions here are better and compensate for the winter conditions in the North.

\section{Availability of Multiple Stations}

If a ground station network has an availability requirement of $M>1$, e.g. to reuse optical frequencies for capacity improvements or to have a continuous backup link, it must be determined how many stations are available at a given time. This can be done by using Equation 17. Figure 13 shows the Link Outage Probability for different availability requirements $M$, assuming the three previously optimized networks with $N=12$ stations.

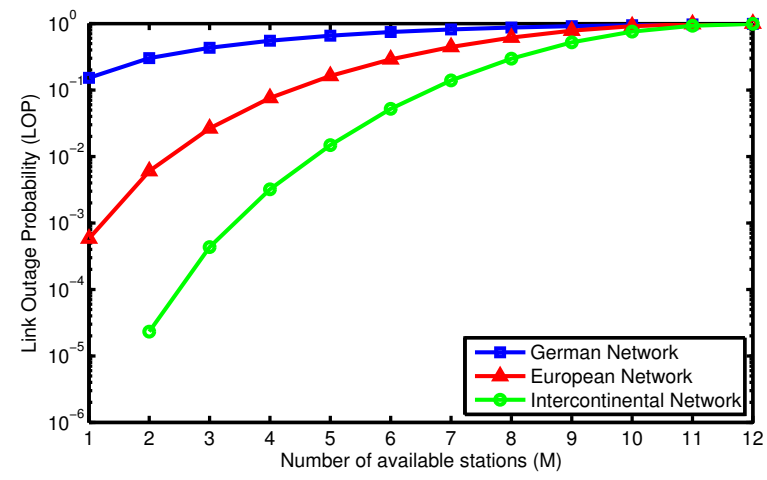

Fig. 13. Link Outage Probability for $N=12$ vs. availability requirement $M$

For instance, in case of the European Network, it can be observed that the Link Outage Probability drops from about $10^{-3}$ to $10^{-2}$ if the availability requirement $M$ is increased from 1 to 2 . Thus, if the continuous availability of two optical links must be given at all times, the number of optical ground stations forming the network must be increased in order to achieve a certain availability level.

\section{Time Behavior}

With the relatively short sampling period of $15 \mathrm{~min}$, the duration and behavior of network outages can be determined. This is of interest for knowing if a reduced Link Outage Probability is due to many short fades with durations in the order of the sampling period, or due to fewer long fades with longer duration, resulting in long network outages.

A histogram for the three networks is presented in Figure 14. It is quite obvious that networks with more stations have shorter outages than smaller networks. It can be seen that the German network has comparatively high outage times. This can be attributed to the high correlation between the ground station sites. Adding further stations to the network, however, would only yield minimal benefits.

Figure 15 shows the probability density function. It reveals that the gradients of the PDFs increase with increasing $\mathrm{N}$. Therefore, larger networks, together with the overall better availability, show a tendency of shorter fades which is advantageous for operations that have particular delay time restrictions.
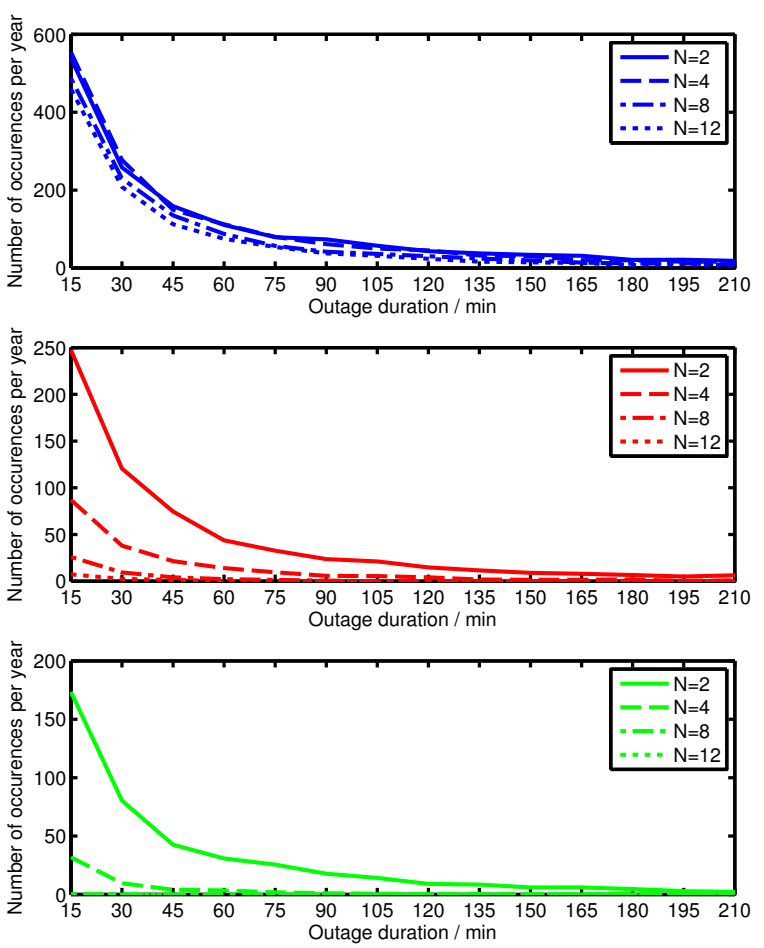

Fig. 14. Histogram of outage durations for different numbers of stations $N$ within the German (top), European (middle), and intercontinental (bottom) networks

Due to the lower correlation between the ground station sites, the European and the intercontinental networks profit most from an increased number of ground stations within their networks.

Figure 16 shows the mean annual outage duration, i.e. the network downtime per year. This is a useful measure for the quality of a ground station network.

\section{CONClusion}

In this paper, we briefly reviewed the calculation of correlated and uncorrelated availability for optical ground station networks in the scenario of space-to-ground optical communication links, with a focus on links to GEO satellites.

An efficient optimization algorithm was developed, enabling reduced computational effort for optimizing optical ground 

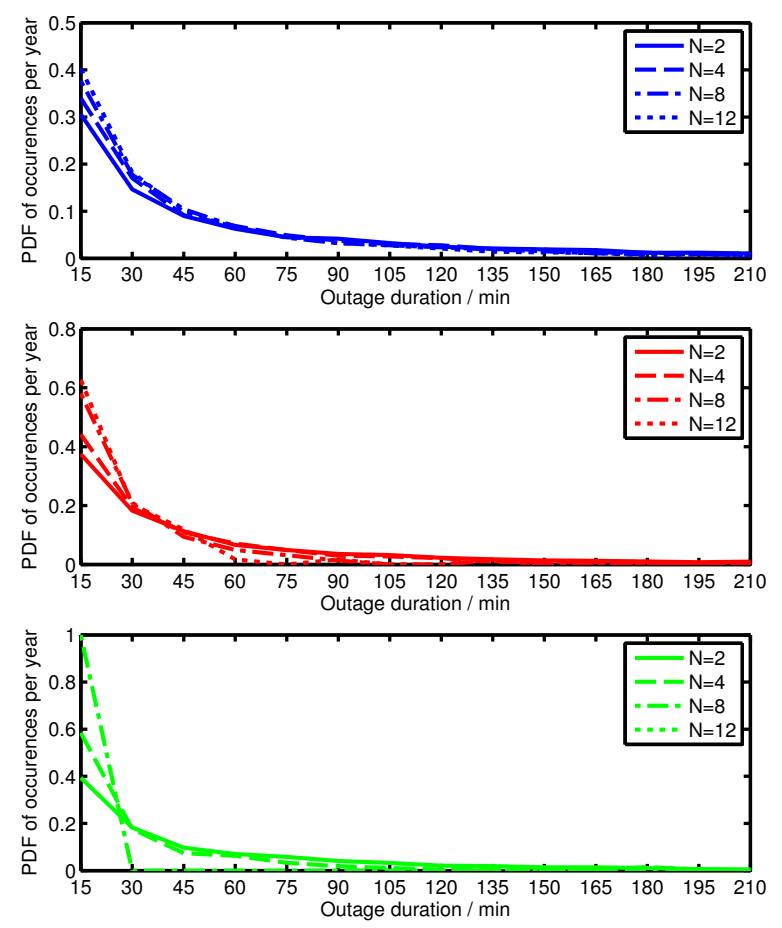

Fig. 15. Probability Density Function (PDF) of outage durations for different numbers of stations $N$ within the German (top), European (middle), and intercontinental (bottom) networks

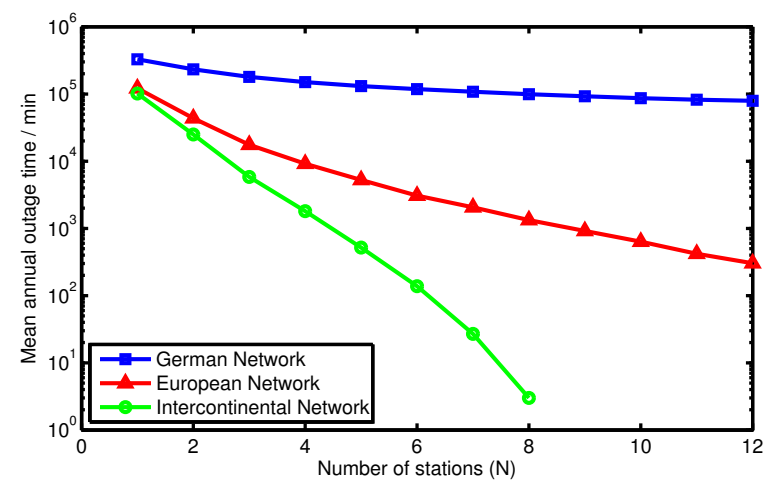

Fig. 16. Mean annual outage duration for different numbers of stations $N$ within the German, European, and intercontinental networks

station networks. It makes use of single-site availability together with correlation between sites. This approach enables finding OGS networks based on a given pool of stations, in our case 66, with low computational effort. The algorithm proved to work well and efficient, however, as a number of OGS sites and combinations are preselected, potentially better combinations might be excluded from further analysis. Thus, future improvements of the algorithm might include an optimized site-preselection, potentially making use of further metrics.

Five years of cloud data were evaluated to simulate the behavior of OGS networks under realistic conditions. Simulation results for three different network topologies (German, European, and intercontinental) were presented and discussed.

The German network suffers from strong correlation be- tween the ground station sites, resulting in limited availability improvements for larger networks. If the number of stations is increased, the Link Outage Probability reaches a value of 0.153 , corresponding to an availability of $84.7 \%$. The European and intercontinental networks show remarkably better results, as, in general, the weather conditions in these networks are better, and the correlation between the ground station sites is reduced. It could be shown that Link Outage Probabilities in the order of $10^{-3}$ can be achieved with a 12 station European network, which results in an availability of about $99.9 \%$. The intercontinental network has been found to have a LOP of $5.8 \cdot 10^{-6}$ for $N=8$, i.e. an availability of $99.999 \%$, or an outage time of only about 5 minutes per year. For $N \geq 9$, the availability of the intercontinental network was even found to be $100 \%$, however, it should be noted that these results are limited to the observation period of 5 years.

Future work might involve the improvement of the OGS database. For optical GEO feeder links, the connectivity to optical fiber ground networks is essential. Reviewing the exact capabilities of each ground station site in the database and adding further stations with good network connectivity will enable more practical results. However, it is not expected that the general trend in the evaluations would be different. Furthermore, the analysis could benefit from a larger cloud database, i.e. using 10 or 15 years of cloud data instead of 5 .

\section{APPENDiX A}

\section{GRound STATION SITE DATABASE}

Tables V, VI, and VII show the OGS sites that have been selected for the study. The Single Station Availability $\left(p_{i}\right)$ was calculated and is presented in the tables as well.

Please note that the values for $p_{i}$ of Izana and La Palma (Table VI) are much lower than expected when using other databases. This is likely due to the fact that MSG2 cloud mask data do not suitably reflect the mountain top location of these sites, with clouds lingering below the actual station location. Thus, Izana and La Palma unfortunately had to be excluded.

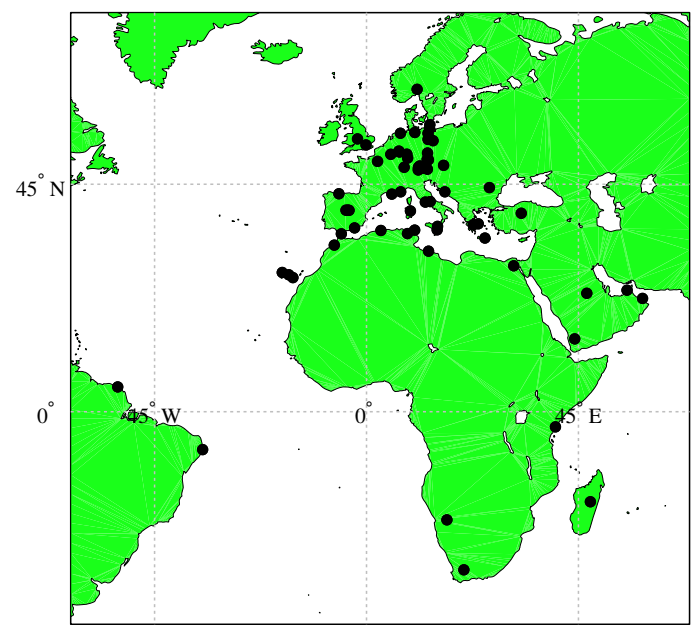

Fig. 17. OGS database that was used for the network optimizations. The OGS sites listed in Table V are marked with a black dot. 


\begin{tabular}{rllrrr}
\hline$i$ & Category & Name & Lat. & Lon. & $\mathrm{p}_{\mathrm{i}}$ \\
\hline 1 & Germany & Berlin & 52.52 & 13.40 & 31.6 \\
2 & Germany & Oberpfaffenhofen & 48.08 & 11.28 & 31.2 \\
3 & Germany & Weilheim & 47.88 & 11.08 & 33.1 \\
4 & Germany & Zugspitze & 47.42 & 10.98 & 36.8 \\
5 & Germany & Wendelstein & 47.70 & 12.01 & 33.2 \\
6 & Germany & Hohenpeißenberg & 47.80 & 11.00 & 34.1 \\
7 & Germany & Lindenberg (Brandenburg) & 52.20 & 14.13 & 30.2 \\
8 & Germany & Potsdam & 52.40 & 13.06 & 31.3 \\
9 & Germany & Effelsberg (Bonn) & 50.53 & 6.88 & 26.1 \\
10 & Germany & Königstuhl (Heidelberg) & 49.40 & 8.72 & 30.3 \\
11 & Germany & Wettzell & 49.14 & 12.88 & 31.2 \\
12 & Germany & Hanburg-Bergedorf & 53.48 & 10.24 & 28.1 \\
13 & Germany & Neustrelitz & 53.35 & 13.05 & 29.4 \\
14 & Germany & Frankfurt (Main) & 50.03 & 8.57 & 30.5 \\
15 & Germany & Watzmann (Mountain) & 47.56 & 12.92 & 30.1 \\
16 & Germany & Großer Arber (Mountain) & 49.11 & 13.13 & 29.9 \\
17 & Germany & Fichtelberg (Ore Mountains) & 50.22 & 12.95 & 26.1 \\
18 & Germany & Emden & 53.37 & 7.20 & 29.8 \\
19 & Germany & Greifswald & 54.08 & 13.38 & 27.8 \\
20 & Germany & Kap Arkona (Rügen island) & 54.68 & 13.44 & 30.7 \\
21 & Germany & Feldberg (Black Forest) & 47.87 & 8.00 & 30.1 \\
22 & Germany & Hohe Acht (Eifel) & 50.39 & 7.01 & 25.1 \\
\hline
\end{tabular}

TABLE V

PRE-SElected GeRmAn OGS Sites.

\begin{tabular}{rllrrr}
\hline$i$ & Category & Name & Lat. & Lon. & $\mathrm{p}_{\mathrm{i}}$ \\
\hline 50 & World & Tunis & 36.84 & 10.24 & 47.1 \\
51 & World & Windhoek & -22.57 & 17.08 & 73.8 \\
52 & World & Sutherland & -32.40 & 20.67 & 55.0 \\
53 & World & El Kef & 36.18 & 8.70 & 59.0 \\
54 & World & Dubai & 25.27 & 55.30 & 66.1 \\
55 & World & Kourou & 5.25 & -52.80 & 47.8 \\
56 & World & Malindi & -3.21 & 40.10 & 67.3 \\
57 & World & Recife & -8.03 & -34.80 & 61.6 \\
58 & World & Cairo & 30.06 & 31.24 & 80.4 \\
59 & World & Tripoli & 32.88 & 13.19 & 75.8 \\
60 & World & Ankara & 39.91 & 32.86 & 53.2 \\
61 & World & Algier & 36.76 & 3.05 & 53.8 \\
62 & World & Rabat & 34.01 & -6.84 & 71.0 \\
63 & World & Antananarivo & -18.90 & 47.52 & 44.7 \\
64 & World & Riad & 24.64 & 46.77 & 66.5 \\
65 & World & Sanaa & 15.35 & 44.21 & 71.2 \\
66 & World & Muscat & 23.61 & 58.59 & 61.9 \\
\hline
\end{tabular}

TABLE VII

PRE-SELECTED INTERCONTINENTAL OGS SITES

\section{ACKNOWLEDGMENT}

The authors would like to thank Prof. Erich Lutz for helpful discussions related to this work, as well as EUMETSAT for providing the cloud data used within this study.

\section{REFERENCES}

[1] J. Horwath and C. Fuchs, "Aircraft to ground unidirectional laser-comm. terminal for high resolution sensors," in Proc. SPIE, 7199. Free-Space Laser Communication Technologies XXI, San Jose, USA, 2009.

[2] F. Moll, W. Mitzkus, J. Horwath, A. Shrestha, M. Brechtelsbauer, L. Martin Navajas, A. Lozano Souto, and D. Diaz Gonzales, "Demonstration of high-rate laser communications from fast airborne platform: flight campaign and result," in Proc. of SPIE, 9248. Unmanned/Unattended Sensors and Sensor Networks X, 22-25 Sep. 2014, Amsterdam, Netherlands, 2014.

[3] D. M. Boroson, B. S. Robinson, D. V. Murphy, D. A. Burianek, F. Khatri, J. M. Kovalik, Z. Sodnik, and D. M. Cornwell, "Overview and results of the lunar laser communication demonstration," in Proc. of SPIE, vol. 8971, 2014.

[4] C. Schmidt, M. Brechtelsbauer, F. Rein, and C. Fuchs, "OSIRIS payload for DLR's BiROS satellite," in Proc. of International Conference on Space Optical Systems and Applications (ICSOS), Kobe, Japan, 2014.

[5] M. Witting et al., "Status of the european data relay satellite system," in Proc. International Conference on Space Optical Systems and Applications (ICSOS), Ajaccio, Corsica, France, 2012.

[6] E. Ciaramella, Y. Arimoto, G. Contestabile, M. Presi, A. D'Errico, V. Guarino, and M. Matsumoto, "1.28 terabit/s (32x40 gbit/s) wdm transmission system for free space optical communications," Selected Areas in Communications, IEEE Journal on, vol. 27, no. 9, pp. 16391645, December 2009.

[7] G. Parca, A. Shahpari, V. Carrozzo, G. M. Tosi Beleffi, and A. L. J. Teixeira, "Optical wireless transmission at 1.6-tbit/s (16x100gbit/s) for next-generation convergent urban infrastructures," Optical Engineering, vol. 52 , no. $11,2013$.

[8] E. Lutz, "Achieving a terabit/s geo satellite system," in 19th $\mathrm{Ka}$ and Broadband Communications, Navigation and Earth Observation Conference, Florence, Italy, 2013.

[9] F. Moll and M. Knapek, "Wavelength selection criteria and link availability due to cloud coverage statistics and attenuation affecting satellite, aerial, and downlink scenarios," in Proceedings of SPIE, vol. 6709, 2007.

[10] J. Horwath, N. Perlot, M. Knapek, and F. Moll, "Experimental verification of optical backhaul links for highaltitude platform networks: Atmospheric turbulence and downlink availability," Int. J. Satell. Commun. Network, vol. 25, pp. 501-528, 2007.

[11] J. H. Churnside and K. Shaik, "Atmospheric propagation issues relevant to optical communications," NOAA, Boulder, Colorado, NOAA Technical Memorandum ERL WPL-159, 1989. 
[12] F. Lacoste, A. Guerin, A. Laurens, G. Azema, C. Periard, and D. Grimal, "Fso ground network optimization and analysis considering the influence of clouds," in Proceedings of the 5th European Conference on Antennas and Propagation, 2011.

[13] G. S. Wojcik, H. L. Szymczak, R. J. Alliss, R. P. Link, M. E. Craddock, and M. L. Mason, "Deep-space to ground laser communications in a cloudy world," in Proceedings of SPIE, vol. 5892, 2005.

[14] R. J. Allis and B. Felton, "The mitigation of atmospheric impacts on free-space optical communications," in Proceedings of ICSOS 2012, Ajaccio, Corsica, France, 2012.

[15] A. Biswas, C.-C. Chen, S. J. Dolinar, W. H. Farr, A. A. Gray, J. Hamkins, H. Hemmati, C.-W. Lau, S. Lee, J. R. Lesh, B. E. Moision, G. G. Ortiz, S. Piazzolla, W. T. Roberts, M. Srinivasan, V. A. Vilnrotter, K. E. Wilson, and M. W. Wright, Deep Space Optical Communications, H. Hemmati, Ed. Hoboken: Wiley \& Sons, 2006.

[16] Y. Takayama, M. Toyoshima, and N. Kura, "Estimation of accessible probability in a low earth orbit satellite to ground laser communications," Radioengineering, vol. 19, pp. 249-253, 2010.

[17] Y. Takayama, Y. Koyama, H. Takenaka, H. Kunimori, and M. Toyoshima, "Studies on candidate approaches for satelliteground laser communications," in Proc. International Conference on Space Optical Systems and Applications (ICSOS) 2012, 2012.

[18] S. Poulenard, B. Roy, M. Hanna, H. L. G. Lacoste, and A. Rissons, "Optical ground station network optimization and performances for high data rate geosatellite-to-ground telemetry," in Proceedings of 6th ESA International Workshop on Tracking, Telemetry and Command Systems for Space Applications, 2013.

[19] S. Poulenard, M. Ruellan, B. Roy, J. Riedi, F. Parol, and A. Rissons, "High altitude clouds impacts on the design of optical feeder link and optical ground station network for future broadband satellite services," in Proceedings of SPIE, vol. 8971, 2014.

[20] D. H. Bromwich, J. P. Nicolas, K. M. Hines, J. E. Kay, E. L. Key, M. A. Lazzara, D. Lubin, G. M. McFarquhar, I. V. Gorodetskaya, D. P. Grosvenor, T. Lachlan-Cope, and N. P. M. van Lipzig, "Tropospheric clouds in antarctica," Review of Geophysics, vol. 50, 2012.

[21] T. Dreischer, M. Tuechler, K. Kudielka, G. Baister, and D. G. und Florian Moll, "Enhanced data return from lunar farside using rf/optical tt\&c," in To Moon and beyond - DGLR International Symposium, 2008.

[22] N. Perlot and J. Perdigues Armengol, "Model-oriented availability analysis of optical geo-ground links," in Proc. SPIE, 8246. Free-Space Laser Communication Technologies XXIV, San Francisco, USA, 2012.

[23] D. M. A. Aminou, B. Jacquet, and F. Pasternak, "Characteristics of the meteosat second generation $(\mathrm{msg})$ radiometer/imager: Seviri," in Sensors, Systems, and Next-Generation Satellites, vol. 3221, 1997, pp. 19-31.

[24] M. Derrien and H. Le Gléau, "SAFNWC/MSG SEVIRI CLOUD PRODUCTS," Proc. of EUMETSAT Meterological Satellite Conference, Weimar, Germany, vol. 29, pp. 191-198, 2003.

[25] — - "MSG/SEVIRI cloud mask and type from SAFNWC," International Journal of Remote Sensing, vol. 26, pp. 4707-4732, 2005.

Christian Fuchs graduated in Telecommunications / Electronics from the University of Applied Sciences, Mannheim, Germany in 2006. Since then he has been with DLR's Institute of Communications and Navigation and coordinated several national and international research projects regarding freespace optical communications in aeronautical- and pace-environments. Since 2011, he has been leading the Optical Communication Systems Group. His research interests include optical communication systems for aeronautical applications, LEO-downlinks, as well as for GEO-feeder links.

Florian Moll Florian Moll received his Dipl. Ing (FH) in electrical engineering from the Jena University of Applied Sciences in 2006 and a M.Sc. degree in electrical engineering from the Technische Universitaet Muenchen (TUM) in 2009. He has been a member of the German Aerospace Centre (DLR), Institute of Communications and Navigation since 2009 and is member of the research group Optical Communications Systems. Here, he is in charge of the Optical Ground Station Oberpfaffenhofen. His main research interests are atmospheric turbulence and optical communications in LEO and aeronautical scenarios. 